\title{
Vacinação contra influenza em idosos no Brasil no período de 2016 a 2020
}

\author{
Influenza vaccination in elderly in Brazil in the period from 2016 to 2020 \\ Vacunación contra la influenza en ancianos en Brasil en el período de 2016 a 2020
}

Tanise Nazaré Maia Costa

ORCID: https://orcid.org/0000-0003-1319-5591 Centro Universitário do Estado do Pará, Brasil

E-mail: tanise.costa@prof.cesupa.br

Laura Correa de Souza

ORCID: https://orcid.org/0000-0002-2315-610X

Centro Universitário do Estado do Pará, Brasil

E-mail: lauracorrea_souza@hotmail.com

Júlia Correa Campos de Souza

ORCID: https://orcid.org/0000-0002-8021-3844

Centro Universitário do Estado do Pará, Brasil

E-mail: j_correa@outlook.com

Lucas Afonso Maia Ferreira

ORCID: https://orcid.org/0000-0002-6956-6331

Centro Universitário do Estado do Pará, Brasi

E-mail: lucasafonsomaiaferreira@ gmail.com

Rafaela Seixas Pinho

ORCID: https://orcid.org/0000-0002-3572-0919

Centro Universitário do Estado do Pará, Brasil

E-mail: pinhorafaela15@gmail.com

Gabriel Aranha Sousa Maués

ORCID: https://orcid.org/0000-0003-3526-7269

Centro Universitário do Estado do Pará, Brasil

E-mail: maues39@gmail.com

Paola Bitar de Mesquita Abinader

ORCID: https://orcid.org/0000-0002-8408-2343

Centro Universitário do Estado do Pará, Brasil E-mail: polabitar@gmail.com

Natalia Francisca Damasceno Rodrigues ORCID: https://orcid.org/0000-0003-4135-4799 Centro Universitário do Estado do Pará, Brasil E-mail: natalia.franciscadr@gmail.com

\begin{abstract}
Resumo
Essa pesquisa objetivou avaliar a distribuição geográfica das doses aplicadas da vacina contra a influenza na população com 60 anos ou mais, no período de 2016 a 2020. O método foi a partir de um estudo transversal com consulta do banco de dados do SUS - DATASUS, evidenciando total de quase 7 milhões de doses aplicadas da vacina contra influenza no período do estudo, 1,4 milhões foram para a população idosa, correspondendo a $20 \%$ do total de vacinados nesse período. $\mathrm{O}$ ano em que foi registrado o maior número de aplicações da vacina na população idosa foi o ano de 2016 e o menor número foi em 2018, sendo que nos anos seguintes houve aumento. Em relação à distribuição geográfica, observou-se maior número de vacinados na região sudeste. A região com menor número de doses aplicadas foi a região centro-oeste. Conclui-se que, apesar da diminuição observada do número de doses aplicadas no decorrer do período estudado, ainda se encontra maior concentração das doses aplicadas na região sudeste. Logo, torna-se necessária a disseminação de mais assistência de saúde e de informação para facilitar o acesso da população idosa à vacinação.
\end{abstract}

Palavras-chave: Idoso; Cobertura vacinal; Prevenção primária.

\begin{abstract}
This research aimed to evaluate the geographic distribution of applied portions of the influenza vaccine in the citizens aged 60 years or more, in the period from 2016 to 2020. The method was based on a cross-sectional study with consultation of the SUS database - DATASUS, evidencing a total of almost 7 million applied doses of the influenza vaccine during the study period, 1.4 million were for the elderly population, corresponding to $20 \%$ of the total vaccinated in that period. The year in which the large number of vaccine applications in the elderly population was recorded was 2016 and the smallest number was in 2018, with an enlarged in the backing years. Regarding geographic distribution, there was a greater number of vaccinated in the Southeast region. The region with the lowest number of doses applied was the Midwest region. It is concluded that, despite the observed decline in the quantity of doses
\end{abstract}


applied over the period studied, there is still a greater concentration of doses requested in the Southeast region. Therefore, the dissemination of more health and information services is necessary to facilitate the access of the elderly population to vaccination.

Keywords: Aged; Vaccination coverage; Primary prevention.

\section{Resumen}

Esta investigación tuvo como objetivo evaluar la distribución geográfica de las dosis aplicadas de la vacuna antigripal en la población de 60 años o más, en el período de 2016 a 2020. El método se basó en un estudio transversal con consulta de la base de datos del SUS, DATASUS, evidenciando un total de casi 7 millones de dosis aplicadas de la vacuna antigripal durante el período de estudio, 1,4 millones fueron para la población anciana, lo que corresponde al $20 \%$ del total vacunado en ese período. El año en el que se registró el mayor número de aplicaciones de vacunas en la población anciana fue 2016 y el menor en 2018, con un incremento en los años siguientes. En cuanto a la distribución geográfica, hubo un mayor número de vacunados en la región sudeste. La región con el menor número de dosis aplicadas fue la región del medio oeste. Se concluye que, apesar de la disminución observada en el número de dosis aplicadas durante el período estudiado, aún existe una mayor concentración de dosis aplicadas en la región sudeste. Por ello, es necesario difundir más servicios de salud e información para facilitar el acceso de la población anciana a la vacunación.

Palabras clave: Anciano; Cobertura de vacunación; Prevención primaria.

\section{Introdução}

No Brasil, é revelado um crescente envelhecimento populacional, ratificado conforme o Censo Demográfico de 2010 do Instituto Brasileiro de Geografia e Estatística (IBGE), no qual a população constava um total de 190,7 milhões de pessoas, sendo 20,5 milhões de idosos, correspondendo à 10,8\% do total; e em 2012, os indivíduos com 60 anos ou mais eram 25,4 milhões. Os 4,8 milhões de novos idosos correspondem a um crescimento de $18 \%$ desse grupo etário, que tem se tornado cada vez mais representativo. Adentrando no ano de 2017, alcançou 30,2 milhões, conforme a Pesquisa Nacional por Amostra de Domicílios Contínua (IBGE, 2017).

Destarte, o país atravessa também o processo de transição epidemiológica, o qual acompanha o envelhecimento populacional, que segundo prospecções do IBGE, a população dos mais velhos irá ultrapassar a de jovens, mudando a pirâmide etária brasileiro no ano de 2050, tornando um dos países com a população mais envelhecida do mundo (Carvalho \& Rodríguez-Wong, 2008). Associado a isto, ocorre o aumento da incidência e prevalência de doenças crônicas e degenerativas e devido a essa nova realidade é necessário ações preventivas visando o aumento da qualidade de vida dos mais anciões (Oliveira, 2019).

Logo, com a visibilidade desse grupo populacional, há peculiaridades em seu cuidado, pois existem síndromes geriátricas, como a insuficiência cognitiva, incontinência urinária, iatrogenia, instabilidade postural e imobilidade (Vilela, Moraes \& Lino, 2008), os quais reduzem significativamente a capacidade dos mais velhos para realizar suas atividades básicas e instrumentais da vida diária, traduzindo em grande declínio funcional, social e pessoal caso não sejam prevenidos e evitados (Costa Filho et al., 2018).

Devido a essa maior vulnerabilidade biológica, um dos grandes métodos de prevenção primária é a vacinação, sendo que a vacina contra o vírus da influenza já está no calendário vacinal do idosos pelo Ministério da Saúde (MS) e desde sua implantação demonstrou significativa redução dos casos de internação, mortalidade e contribui para o avanço e melhoria dos índices de qualidade de saúde pública (Silva et al., 2021; Moura et al., 2015; Aoyama et al., 2019).

Apesar do supracitado, a adesão da população idosa a vacinação ainda é insatisfatória. Fatores como escolaridade, condições socioeconômicas, laborais, atividade física, número de consultas médicas no último ano interferem no alcance vacinal dos anciões (Azambuja et al., 2020). Ademais, também essa má aderência também decorre a falta de esclarecimento sobre a vacina e suas possíveis complicações, além da desconfiança na vacina com culturas enganosas e conceitos falsos (Monteles et al., 2017). 
Vale ressaltar que essa crença incorreta a respeito de vacinas em geral, deve-se ao fato de que a eficácia da vacinação em idosos depende da resposta imune do indivíduo, da imunogenicidade do imunizante, da combinação antigênica entre a vacina e as cepas circulantes, da saúde do paciente, da cobertura de homogeneidade da vacinação e de fatores externos. E essa prerrogativa contribui para as dúvidas da efetividade do imunizante e a não adesão (Soares et al., 2021).

Dados epidemiológicos denotam que a vacina garante a proteção apropriada, devendo ser administrada anualmente. E apesar da eficiência do imunizante, ainda ocorrem óbitos anuais por H1N1, muitas vezes porque nem toda a população teve acesso à vacina (Côrtes et al., 2020).

Dessa forma, o presente estudo propõe observar a distribuição geográfica das doses aplicadas da vacina contra influenza referente a população de 60 anos ou mais no período de 2016 a 2020 nas diferentes regiões brasileiras.

\section{Metodologia}

Trata-se de um estudo transversal e descritivo, epidemiológico e documental, retrospectivo, com abordagem quantitativa feita através de pesquisa no conjunto de informações provenientes do Departamento de Informática do Sistema Único de Saúde (DATASUS), com o objetivo de analisar a vacinação contra influenza em idosos, por esse motivo, foram usados os descritores de Assistência em Saúde, do TABNET, na população com faixa etária maior que 60 anos e no período compreendido de 2016 a 2020.

A pesquisa se iniciou com obtenção do banco de dados e posterior análise e descrição dos mesmos. Os dados coletados foram pertinentes às cinco regiões do Brasil (Centro-oeste, Sudeste, Sul, Norte e Nordeste). Foram incluídos neste estudo todos os dados de casos que foram notificados no referente período. Excluiu-se dados registrados fora do contexto do estudo.

Considerando as características da pesquisa, os riscos foram mínimos pois os dados foram coletados diretamente no banco de dados oficial do SINAN, sendo garantida a confidencialidade das informações, a privacidade e o anonimato dos participantes para evitar prejuízo. Os benefícios foram indiretos, e associados ao levantamento de informações que ajudam a redirecionar ações.

O Banco de dados foi baseado na plataforma digital DATASUS. Após, foram organizados em planilhas do aplicativo Excel e submetidos a análise estatística pelo programa Bio-Estat 5.0.

\section{Resultados}

Este estudo observou total aproximadamente 7 milhões de doses aplicadas da vacina contra influenza no período de 2016 a 2020; destes, pouco mais de 1,4 milhões foram na população idosa, correspondendo a 20\% do total de vacinados nesse período. 
Research, Society and Development, v. 10, n. 14, e235101421909, 2021

(CC BY 4.0) | ISSN 2525-3409 | DOI: http://dx.doi.org/10.33448/rsd-v10i14.21909

Figura 1: Distribuição das doses aplicadas da vacina influenza.

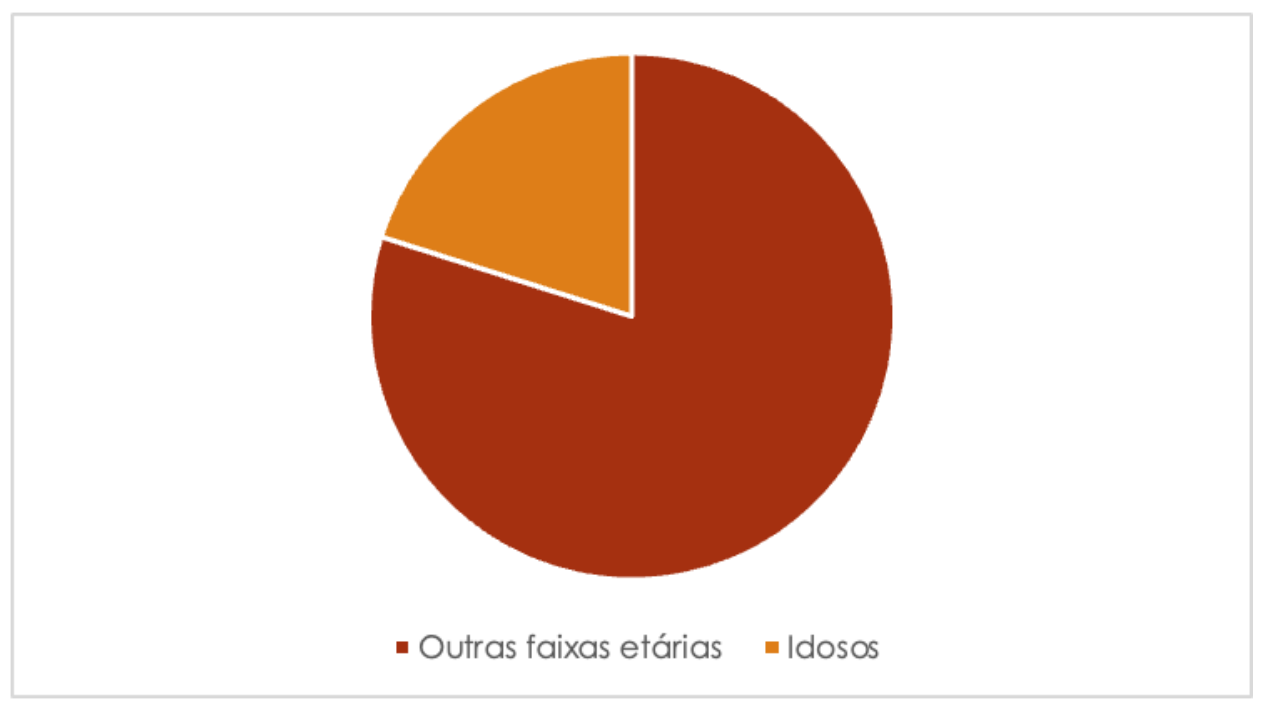

Fonte: DATASUS.

Gráfico 1: Número de doses da vacinação contra influenza aplicadas em idosos entre o ano de 2016-2020.

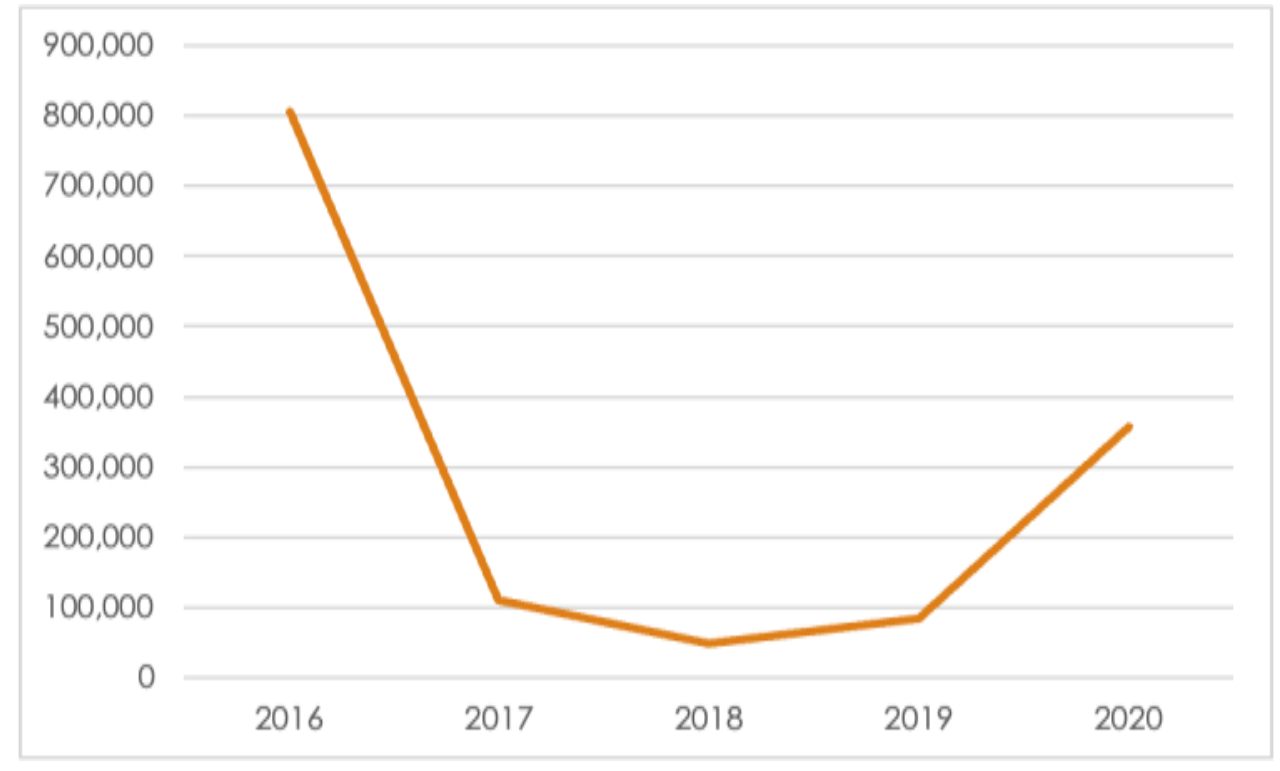

Fonte: DATASUS. 
Gráfico 2: Amostra de doses da vacina contra influenza aplicadas em idosos, de acordo com mesorregiões brasileiras, no período de 2016-2020.

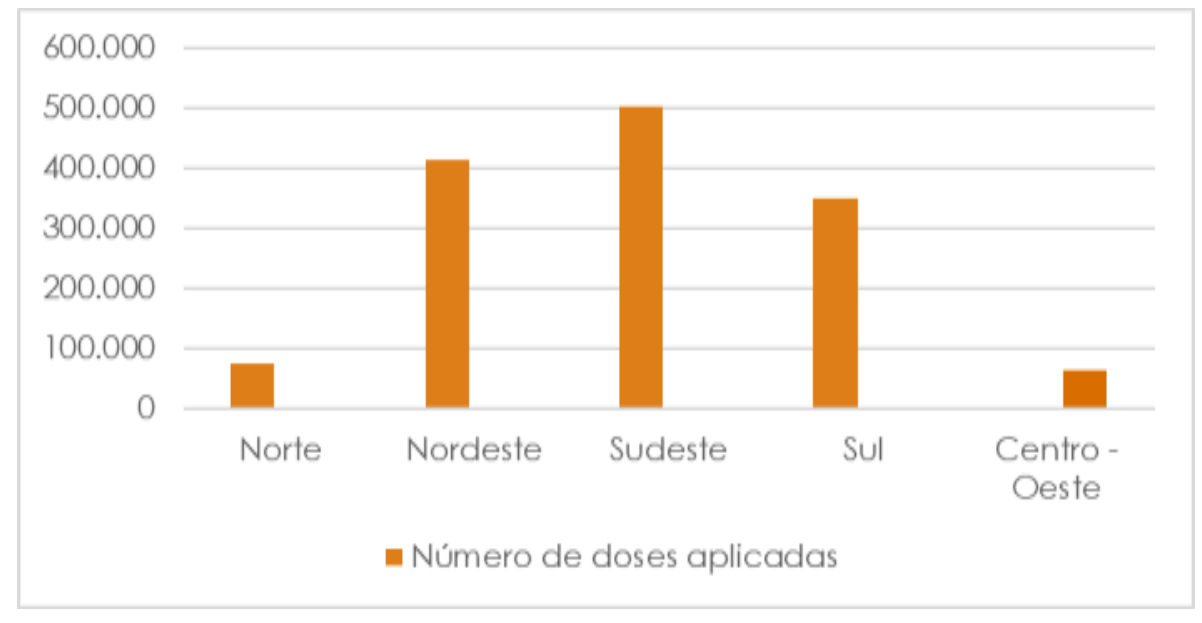

Fonte: DATASUS.

\section{Discussão}

Historicamente, em 1999, a população brasileira alvo de vacinação era, exclusivamente, o público acima de 65 anos segundo o Informe Técnico na 21 a Campanha Nacional de Vacinação contra a Influenza (2019). Porém, a partir de 2011, foram adicionados novos grupos etários, e, portanto, a população idosa, que antes era a totalidade a receber as doses produzidas e administradas, passou a ter uma nova porcentagem populacional reduzida em termos quantitativos de imunização na população total.

Essa informação é importante pois permite compreender, de acordo com dados coletados entre 2016-2020, a porcentagem encontrada no trabalho que foi em torno de $20 \%$ das imunizações serem em idosos, pois atualmente, não são mais administradas em sua universalidade neste grupo etário.

Levando em consideração o acesso vacinal especificamente contra esse vírus apenas na população geronte, segundo o estudo de Azambuja et al (2020) elevou-se bastante nos últimos tempos, alcançando valores próximos ou superiores a 100\% em 2019. Apesar disso, é notável que a vacina contra a gripe ainda não englobou os idosos brasileiros de forma completa, porque há variabilidade da cobertura no discorrer dos anos e de acordo com a região do país.

Os resultados do presente estudo também demonstram que as pessoas idosas apresentaram redução do número de imunização de 2017 a 2018, seguido de aumento gradativo de 2019 até 2020. Provável explicação para essa elevação pode ser que no ano de 2020 a pandemia pelo Covid-19 estava em pleno auge e tal fato pode ter corroborado com as medidas de incentivo a vacinação e a maior procura da população por imunizar contra influenza para não ser o quadro gripal causado por este vírus confundido com o provocado pelo novo coronavírus, já que ambos se assemelham em alguns aspectos (Lana et al., 2020).

Em contraponto, imunizações de rotina durante pandemias podem ter sido prejudicadas como demonstrou o estudo de Menezes et al. (2021) que calculou a cobertura vacinal de 82,3\% para influenza em idosos durante a COVID-19 através do EPICOVID-19.

Em relação a diminuição de notificação de dados em 2018, é concordante com o trabalho de Cabral \& Gonçalves, em 2020, que discorre acerca da diminuição no município da região sul Fluminense do número absoluto de idosos vacinados em 2018, assim como a meta de vacinação definição pelo Ministério da Saúde, a qual teve relação direta com o aumento na taxa de internação por pneumonia no município. 
No que se refere a comparação entre as regiões brasileiras, o maior número de vacinação no Sudeste está relacionado ao maior desenvolvimento socioeconômico (Sato et al., 2020). E, apesar da região centro-oeste ter o menor número de vacinações aplicadas na análise desta pesquisa, o contrário pode ser evidenciado no que tange ao Distrito Federal, geralmente a maior taxa, devido ao centro político da área. Esses dados conciliaram com o estudo de Azambuja et al (2020), o qual refere aumento da taxa de vacinação a partir de 2016, com exceção da região Centro-Oeste.

No entanto, em um estudo não comparativo interrregiões de Silva et al. (2021), e sim isoladamente no Centro-oeste, constatou a cobertura vacinal anti-influenza nesta região de 95,6\% e entre os 53 municípios, a maioria (84,9\%) alcançou a meta de cobertura vacinal de influenza de 90\%, conforme preconizado pelo MS. Essas constatações são reforçadas diante da problemática heterogeneidade entre estados brasileiros em relação ao alcance vacinal em habitantes senis com a vacina antiinfuenza (Victor et al., 2014).

De alguma forma, de posse dessas informações, é necessário explicitar a importância do papel do trabalhador da saúde em orientar e esclarecer todos os pontos da vacina para aumentar a adesão às mesmas com intuito de sistematicamente manter níveis iguais ou superiores em termos de cobertura vacinal estipulada pelo MS.

\section{Conclusão}

Salienta-se que, apesar da diminuição do número de doses aplicadas no decorrer do período estudado, ainda se observa adesão da população estudada às campanhas de vacinação contra influenza, já que há uma crescente na curva vacinal dos idosos, apesar de diversidade entre as regiões, como por exemplo maior concentração das doses aplicadas na região sudeste, podendo ser usada como molde para demais localidades, ao se tratar de políticas públicas e educação em saúde.

Logo, torna-se necessária a disseminação de mais acesso à saúde e de informação para facilitar e estimular o acesso da população idosa à vacinação, para que assim a crescente permaneça, melhorando reduzindo os índices epidemiológicos de morbimortalidade e a qualidade de vida desse grupo etário.

\section{Referências}

Andrade, A. B. C. A. de, Albuquerque, B. C. de, Garnelo, L., \& Herkrath, F. J. (2021). Vacinação contra a influenza autorreferida por idosos de áreas rurais ribeirinhas: implicação potencial dos achados frente à pandemia de covid-19 no Amazonas. Revista Brasileira de Geriatria e Gerontologia, 24(3):e210094.

Aoyama, E. de A., Nunes, E. C. A., Oliveira, M. da S., Silva, S. L. da, Araújo, J. A. F. de, \& Firmino, T. de, A. B. (2019). Os benefícios da vacina H1N1 em idosos. Brazilian Journal of. Health Review, 2 (1), 185-91.

Azambuja, H. C. S., Carrijo, M. F., Martins, T. C. R., \& Luchesi, B. M. (2020). O impacto da vacinação contra influenza na morbimortalidade dos idosos nas regiões do Brasil entre 2010 e 2019. Cadernos de Saúde Pública, 36, e00040120. https://doi.org/10.1590/0102-311X00040120

Brasil, Ministério da Saúde. Banco de dados do Sistema Único de Saúde-DATASUS. http://www.datasus.gov.br

Cabral, M. D., Gonçalves, S. J. C. (2020). Estudo retrospectivo das internações hospitalares por pneumonia X cobertura vacinal para influenza A em pessoas acima de 60 anos de idade. Revista de Saúde. 11 (2), 10-4.

Carvalho, J. A. M de., \& Rodríguez-Wong, L. L. A. (2008). Transição da estrutura etária da população brasileira na primeira metade do século XXI. Cadernos de Saúde Pública. 24 (3), 597-605.

Cortêz, T. H. C., Pinto, I. S., Cortêz, A. H. da S., Bastos, K. . Z. C., \& Britto, M. H. R. M. (2020). Evaluation of the epidemiological profile of vaccination against the H1N1 virus in the state of Piauí, Brazil. Research, Society and Development, 9(10), e3729108626. https://doi.org/10.33448/rsd-v9i10.8626

Costa Filho, A. M. et al. (2018). Contribuição das doenças crônicas na prevalência da incapacidade para as atividades básicas e instrumentais de vida diária entre idosos brasileiros: pesquisa nacional de saúde. Cadernos de Saúde Pública, 34 (1).

Fundação Nacional de Saúde. Informe técnico: 21 a Campanha Nacional de Vacinação contra a Influenza 2019. http://www.cosemssp.org.br/wpcontent/uploads/2019/04/Informe-21\%C2\%AA-Campanha-Nacional-de-Vacina\%C3\%A7\%C3\%A3o-contra-a-Influenza-1.pdf

Instituto Brasileiro de Geografia e Estatística. Censo Demográfico de 2010. Rio de janeiro: IBGE; 2010.

Lana, R. M., Coelho, F. C., Gomes, M. F. da C., Cruz O. G., Bastos, L. S., Villela, D. A. M., \& Codeço, C. T. (2020). Emergência do novo coronavírus (SARS-CoV-2) e o papel de uma vigilância nacional em saúde oportuna e efetiva. Cad Saúde Pública. 36(3), e00019620. doi: 10.1590/0102-311X00019620 
Research, Society and Development, v. 10, n. 14, e235101421909, 2021

(CC BY 4.0) | ISSN 2525-3409 | DOI: http://dx.doi.org/10.33448/rsd-v10i14.21909

Menezes, Ana Maria Baptista et al. (2021). Vacinação para influenza em idosos na pandemia COVID-19: estudo de base populacional em 133 cidades brasileiras. Ciência \& Saúde Coletiva [online], 26 (8), 2937-27. https://doi.org/10.1590/1413-81232021268.09382021.

Monteles, M., Aragão, F., Pereira, J., \& Gomes, F. (2017). Fatores de não adesão dos idosos à vacina contra influenza: uma revisão bibliográfica. Revista Uningá Review, 30(3). http://revista.uninga.br/index.php/uningareviews/article/view/2024

Moura, R. F. et al. (2015). Fatores associados à adesão à vacinação anti-influenza em idosos não institucionalizados, São Paulo, Brasil. Cadernos de Saúde Pública, 31 (10), 2157-68.

Oliveira, A. S. 2019. Transição demográfica, transição epidemiológica e envelhecimento populacional no Brasil. Hygeia-Revista Brasileira de Geografia Médica e da Saúde, 15 (32), 69-79.

Sato, A. P. S. et al. (2020). Cobertura vacinal e fatores associados à vacinação contra influenza em pessoas idosas do Município de São Paulo, Brasil: Estudo SABE 2015. Cadernos de Saúde Pública [online]. 36, (Suppl 2), e00237419. https://doi.org/10.1590/0102-311X00237419.

Silva, P. V. de C., Nicoli, L. H. da S., Oliveira, S. H., Tavares, L. O. de M., Guimarães, E. A. de A., \& Oliveira, V. C. de. (2021). Coverage of anti-influenza vaccination in the elderly, Minas Gerais, Brazil. Research, Society and Development, 10(6), e3610615222. https://doi.org/10.33448/rsd-v10i6.15222

Soares, A. C., Furioso, A. C. T., Moraes, C. F., Gomes, L. O., \& Nóbrega, O T. (2021). Vacinação influenza e o coeficiente de mortalidade por doenças respiratórias em idosos no Brasil. Revista Kairós-Gerontologia, 24(2), 65-84. https://doi.org/10.23925/2176-901X.2021v24i2p65-84

Victor, J. F. et al. (2014). Factors associated with vaccination against Influenza A (H1N1) in the elderly. Revista da Escola de Enfermagem da USP. 48 (1), $57-64$.

Vilela, A. L., Moraes, E. N., Lino, V. (2008). Grandes síndromes geriátricas. Envelhecimento e saúde da pessoa idosa. Fundação Oswaldo Cruz, 193-268. 